\title{
Pharmacogenetics and pharmacogenomics in human diseases
}

\author{
Ramón Cacabelos \\ International Center of Neuroscience and Genomic Medicine, EuroEspes Biomedical Research Center, Bergondo 15165, Spain. \\ Correspondence to: Prof. Dr. Ramón Cacabelos, International Center of Neuroscience and Genomic Medicine, EuroEspes \\ Biomedical Research Center, Bergondo 15165, Spain. E-mail: rcacabelos@euroespes.com. \\ How to cite this article: Cacabelos R. Pharmacogenetics and pharmacogenomics in human diseases. J Trans/ Genet Genom \\ 2021;5:133-5. https://dx.doi.org/10.20517/jtgg.2021.07
}

Received: 1 Mar 2021 Accepted: 2 Mar 2021 Available online: 7 Apr 2021

Academic Editor: Ramón Cacabelos Copy Editor: Yue-Yue Zhang Production Editor: Yue-Yue Zhang

Over the past 20 years the gradual maturation of Genomic Medicine has influenced the medical practice in three principal areas: (1) pathogenesis and the understanding of the primary cause of diseases; (2) diagnosis, with the development of biomarkers; and (3) treatment, with the implementation of pharmacogenetic protocols for personalized therapeutics ${ }^{[1]}$. Another important issue was the understanding of the genomic structure and function of the pharmacogenomic machinery in which pathogenic, mechanistic, metabolic, transporter and pleiotropic genes are involved, as well as their epigenetic regulation (DNA methylation, histone/chromatin modifications, and miRNAs) ${ }^{[2]}$.

In parallel to the development of specific biomarkers for early and/or presymptomatic diagnosis associated with the pathogenic mechanisms underlying the onset of a particular disease, the optimization of limited therapeutic resources by means of personalized treatments is fundamental in the clinical practice.

Cardiovascular disorders (25\%-30\%), cancer (20\%-25\%) and brain disorders (10\%-15\%) represent over 70\% of morbidity and mortality in developed countries. Most of these disorders are chronic and require treatments for long periods of time, with the consequent risk for adverse drug reactions (ADRs) and drugdrug interactions (DDIs), since concomitant diseases demand additional treatments ${ }^{[3]}$. In terms of costs, approximately $15 \%-20 \%$ of direct costs are related to pharmacological intervention. 
This special issue of the Journal of Translational Genetics and Genomics, entitled "Pharmacogenetics and Pharmacogenomics of Human Diseases", is a modest attempt to bring together some ideas from different scientists worldwide with a multidisciplinary background and experience in the field of pharmacogenomics and related disciplines.

Obeng et al. ${ }^{[4]}$ show key preparatory steps in establishing pharmacogenetic implementation programs, including (1) selecting the medications, genes and the laboratory; (2) selecting the patients to test; and (3) providing oversight for program implementation. They also emphasize on drug-gene pairs with strong FDA recommendations, involving several drugs (clopidogrel, codeine, tramadol, pegloticase, rasburicase, carbamazepine, oxcarbazepine, abacavir, azathioprine, mercaptopurine, and thioguanide).

Pandey et al. ${ }^{[5]}$ studied the problems with hydroxyurea treatment in sickle cell disease and how to overcome or minimize myelosuppression and DDIs, postulating a mathematical model to challenge interpatient variability, dose optimization, and non-adherence.

Gengivir et al. ${ }^{[6]}$ screened the pharmacogenomics of the immunosuppressive drug mycophenolic acid in kidney transplantation. The Brazilian authors, under the supervision of Rosario Dominguez, analyzed metabolic and transporter genes potentially associated with the pharmacokinetics and pharmacodynamics of mycophenolic acid.

Amadori and Pasquale Striano's group ${ }^{[7]}$ presented a case of postnatal epilepsy with a de novo missense mutation in the KCNQ2 gene (c.1742G > A; Arg581Gln) at 20q13.3, treated with phenobarbital, levetiracetam and phenytoin.

Segui et al.$^{[8]}$ showed evidence on the influence of pharmacogenomic factors on the effects of buprenorphine in the treatment of opioid use disorder, paying special attention to genes encoding opioid receptors (OPRM1, OPRD1, OPRK1) and metabolic genes involved in phase I-II enzyme reactions.

Finally, Lu et al. ${ }^{[9]}$ confronted the historical analysis of suicide with a brief review on this psychiatric disorder which causes $2 \%$ of human mortality. Unfortunately, genomic and pharmacogenomic data on suicidality are scarce and the pathogenesis of suicidal ideation is poorly understood.

Although, at the present time, the pharmacogenomics of most FDA-approved drugs is being systematically analyzed $^{[1]}$, pharmacogenomics, as a clinical discipline, is still far away from being incorporated as a routine in the clinical practice. Furthermore, the implementation of pharmacogenetic procedures is an urgent need in drug development ${ }^{[10]}$ and in minimizing ADRs and DDIs ${ }^{[3]}$. Many more studies are needed to understand the interaction of commonly used drugs with the genome and characterize those genomic variants that are decisive in the efficacy and safety of each drug, without excluding the influence of epigenetics on the pharmacokinetics and pharmacodynamics of medicines.

\section{DECLARATIONS}

\section{Authors' contributions}

The author contributed solely to the article.

\section{Availability of data and materials}

Not applicable. 


\section{Financial support and sponsorship}

None.

\section{Conflicts of interest}

No conflicts of interest declared.

\section{Ethical approval and consent to participate}

Not applicable.

\section{Consent for publication}

Not applicable.

\section{Copyright}

(c) The Author(s) 2021.

\section{REFERENCES}

1. Cacabelos R. World Guide for Drug Use and Pharmacogenomics. EuroEspes Publishing Co.; Corunna, 2012.

2. Cacabelos R. Pharmacoepigenetics. Academic Press/Elsevier; San Diego, 2019.

3. Cacabelos R, Cacabelos N, Carril JC. The role of pharmacogenomics in adverse drug reactions. Expert Rev Clin Pharmacol 2019;12:407-42. DOI PubMed

4. Obeng A, El Rouby N, Liu M, Wallsten R. Important preparatory steps and clinical considerations for pharmacogenetics adoption into practice. J Transl Genet Genom 2020:4. DOI

5. Pandey A, Estepp JH, Ramkrishna D. Hydroxyurea treatment of sickle cell disease: towards a personalized model-based approach. $J$ Transl Genet Genom 2021:5. DOI

6. Genvigir FDV, Cerda A, Hirata TDC, Hirata MH, Hirata RDC. Mycophenolic acid pharmacogenomics in kidney transplantation. $J$ Transl Genet Genom 2020;4:320-55. DOI

7. Amadori E, Brolatti N, Scala M, et al. Precision medicine in early-onset epilepsy: the KCNQ2 paradigm. J Transl Genet Genom 2020;4:278-84. DOI

8. Seguí HA, Melin K, Quiñones DS, Duconge J. A review of the pharmacogenomics of buprenorphine for the treatment of opioid use disorder. J Transl Genet Genom 2020;4:263-77. DOI PubMed PMC

9. Lu DY, Wu HY, Cao S, Che JY. Historical analysis of suicide. J Transl Genet Genom 2020;4:203-9. DOI

10. Cacabelos R. Population-level pharmacogenomics for precision drug development in dementia. Expert Rev Precis Med Drug Dev 2018;3:163-88. 